\title{
Los efectos del covid-19 en la economía de la población de la región Huancavelica, desde un enfoque de la dinámica de sistemas
}

\author{
The effects of covid-19 on the economy of the population of the region \\ Huancavelica, from a systems dynamics approach
}

Sergio Huincho Lapa' (D), Fernando V. Sinche Crispín²

\section{Cómo citar}

Huincho Lapa, S. (2021). Los efectos del covid-19 en la economía de la población de la región Huancavelica, desde un enfoque de la dinámica de sistemas. Socialium, 5(1), 1-22. https://doi.org/10.26490/uncp.sl.2021.5.1.746

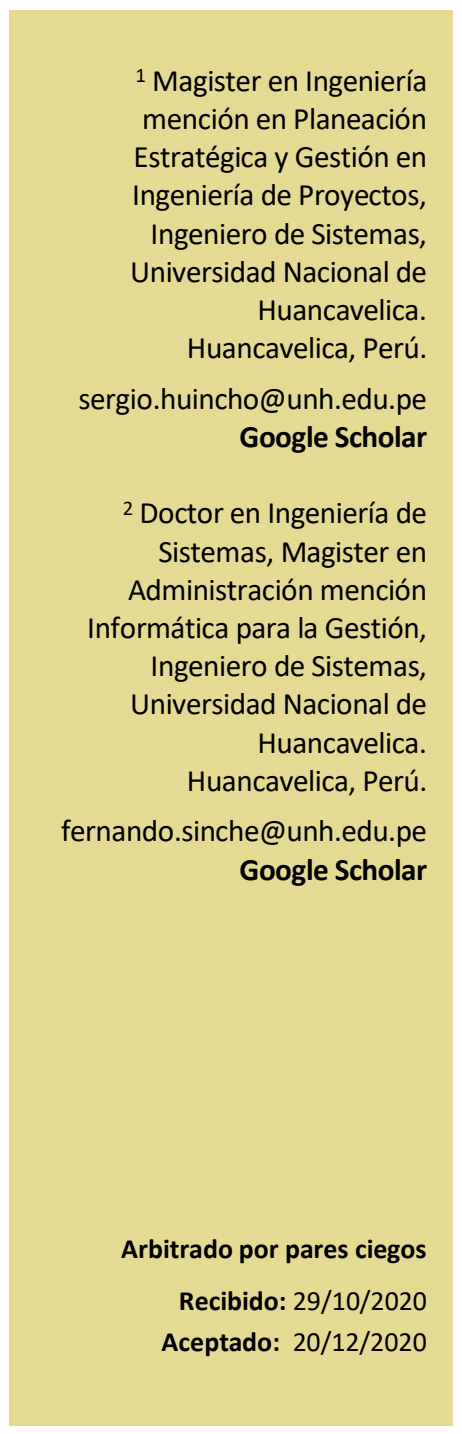

\section{RESUMEN}

La economía de la población rural del Perú, a consecuencia del aislamiento social para controlar los efectos del covid-19, fue la más afectada debido a la caída de la demanda y precio de productos agrícolas, generando pérdida de empleos y empeorando las condiciones de pobreza de la población. Con el objetivo de comprender los efectos de covid19 en la economía de la población y aproximar los efectos al año 2026, se construyó un modelo dinámico. Que permitió analizar la situación en tres escenarios: Primero, corresponde a una situación normal, sin covid-19. Segundo, corresponde a la situación generada por covid-19, y tercero, corresponde a los mecanismos que el gobierno establece para mejorar la situación generada por la pandemia. En conclusión, la pandemia generó la caída de la demanda de productos agrarios hasta $-13 \%$, y los precios hasta $-40 \%$; disminuyendo el empleo hasta $-10 \%$ y aumento de la pobreza hasta más de $10 \%$. Las aproximaciones al año 2026 indican, que la situación empeoraría si no se toma acciones urgentes, y los mecanismos implementados para aumentar la demanda y el precio de los productos agrarios, no tendrían resultados esperados en la recuperación de la economía de la región.

\section{Palabras clave: economía; empleo; modelo; pobreza; pandemia.}

\section{ABSTRACT}

The economy of the rural population of Peru, as a result of social isolation to control the effects of covid-19, was the most affected due to the fall in the demand and price of agricultural products, generating job losses and worsening the conditions of poverty of the population. In order to understand the effects of covid on the economy of the population and approximate the effects to the year 2026, a dynamic model was built. Which allowed us to analyze the situation in three scenarios: First, it corresponds to a normal situation, without covid-19. Second, it corresponds to the situation generated by covid-19, and third, it corresponds to the mechanisms that the government establishes to improve the situation generated by the pandemic. In conclusion, the pandemic generated a fall in the demand for agricultural products to $-13 \%$, and prices to $-40 \%$; reducing employment to $-10 \%$ and increasing poverty to more than $10 \%$. Approaches to the year 2026 indicate that the situation would worsen if urgent actions are not taken, and the mechanisms implemented to increase the demand and price of agricultural products would not have expected results in the recovery of the region's economy.

Keywords: economy; employment; model; poverty; pandemic. 


\section{Introducción}

La pandemia por el covid-19, ha golpeado fuertemente a la economía global, debido a la inmovilización generada por el cierre de fronteras y el aislamiento social obligatorio, decretados por los gobiernos para detener expansión del virus.

La Secretaría de la Comisión Económica para América Latina y el Caribe (CEPAL), advirtió que la América Latina y el Perú no es ajena a esta problemática, y que: la pandemia del covid-19 generaría los efectos devastadores sobre la economía de la región, debido a la caída de la demanda y precio de productos agrarios, como consecuencia de la paralización económica mundial provocando una lamentable pérdida de empleos. Bárcena (2020). Finalmente, estimando, de 620 millones de habitantes que tiene América Latina, el número de pobres pasarían de 185 a 220 millones de personas; en tanto, las personas en pobreza extrema podrían aumentar de 67,4 a 90 millones.

En el Perú, como consecuencia del aislamiento social para combatir el covid-19, hizo que la mayoría de las actividades económicas entraran en paralización, siendo la economía de la población rural como de la región Huancavelica, una de la más afectada, incrementando la situación de pobreza y extrema pobreza. Para León (2020), del Programa de las Naciones Unidas para el Desarrollo PNUD, "más de 2.2 millones de personas se dedican a la agricultura, principalmente en zonas rurales, donde producen el $80 \%$ de los productos que se consumen en las ciudades". Asimismo, Alejandro (2020), de la Comunicación Intercultural Para un Mundo más Humano y Diverso SERVINDI, refiere que, durante la situación de emergencia, la agricultura abasteció los mercados con el 70\% de los productos para la alimentación de más de 30 millones de peruanos. Además, duran-te los primeros meses de emergencia, al mercado mayorista de Lima ingresó 6,573 toneladas de productos en promedio, evidenciando una disminución de $13 \%$ y la tendencia al alza de precio de los productos. Pese a su importancia de la actividad agrícola como generadora de empleos en las zonas rurales, el $88 \%$ de hogares rurales son vulnerables. Toda vez que la actividad carece de un sistema de protección social adecuado, limitaciones al financiamiento y acceso a mercados.

Por otro lado, como señala Berganza (2020), el covid-19 ya viene afectando severamente al empleo, especialmente el de las poblaciones más vulnerables e informales; provocando grandes migraciones, al menos 167000 personas solicitaron ayuda de manera formal a gobiernos locales para salir de las ciudades, y regresar a sus lugares de origen. Generando preocupación sobre una crisis de salud pública en el campo y el aumento del desempleo. 
Finalmente, los datos del censo del 2017 (INEI, 2018), indicaron que: el sector Agricultura, Pesca y Minería abastece el 25.9\% de la PEA con empleo. Sin embargo, para Marco (2020), por causa del covid-19, tendría una caída del $-10 \%$, debido a una reducción de la demanda y precio, de los productos agropecuarios y de los minerales, como consecuencia de las restricciones y el cierre temporal de empresas mineras por disposiciones gubernamentales de emergencia sanitaria.

Esta situación problemática generada por la pandemia requiere una mirada desde el enfoque de sistemas. El comportamiento de los factores económicos revela la esencia sistémica de nuestro mundo, donde los problemas están interconectados y son interdependientes. Observar el contexto aisladamente tendría resultados indeseables. La dinámica de sistemas, un método sistémico para modelar el comportamiento de sistemas complejos sería una herramienta útil para abordar el problema de la pandemia y sus efectos dinámicos.

Por ello, el propósito del presente trabajo es evaluar a través de un modelo dinámico, los efectos de la pandemia del covid-19 en la economía de la población de la región Huancavelica, donde la actividad agrícola es la principal dinamizadora de la economía.

\section{Método}

El trabajo de investigación por los propósitos que persigue se enmarca dentro de la investigación aplicada, y nivel de investigación descriptivo, como refiere Sánchez y Reyes (2017), "la investigación descriptiva se orienta a describir un fenómeno o una situación mediante el estudio del mismo en una circunstancia temporal - espacial determinada" (p. 49). Por lo que, la investigación tiene los propósitos de describir y recoger información sobre los efectos del covid-19 en los aspectos económicos de la población.

El método general de la investigación fue el científico, porque se tomó en cuenta los pasos y procedimientos para la investigación y, el método particular que coadyuvó en el logro de los objetivos deseados fue, el método sistémico, que, consiste a modelar el objeto mediante el enfoque de sistemas, con la determinación de sus elementos y relaciones entre ellas, que establece la estructura del objeto y su dinámica. Como señala Sánchez y Reyes (2017): "el método sistémico tiene como propósito modelar el objeto mediante el estudio de sus partes componentes, así como de las relaciones entre ellos, se investiga por un lado la estructura y por otro lado su desarrollo" (p.54). La Dinámica de Sistemas, un método del enfoque sistémico nos permitió construir el modelo de simulación a través del proceso de modelamiento. Al respecto, Aracil \& Gordillo (1998), considera la Dinámica de Sistemas como, "un método para el estudio 
del comportamiento de sistemas mediante la construcción de un modelo de simulación informática que ponga de manifiesto las relaciones entre la estructura del sistema y su comportamiento" (p.11).

Para la recolección de datos se utilizó el análisis documental que permitió recoger los datos a partir de fuentes secundarias, mediante los instrumentos de formatos y fichas. Los datos recogidos fueron referente a los aspectos económicos de la población, que sirvieron como línea de base para realizar el análisis en tres escenarios. Para determinar la validez y confiabilidad del modelo se utilizó los estadísticos: la media, la desviación estándar y los máximos - mínimos.

\section{Desarrollo del modelo dinámico}

La Región Huancavelica, una de las 24 regiones del Perú ubicada en la sierra central del país, donde la principal fuente de la economía es la actividad agrícola. Los productos agrícolas con mayor superficie en las intenciones de siembra es la papa, la cebada grano y el maíz amiláceo; los mismos tienen el alcance de exportación a los diferentes mercados nacionales.

\section{La situación antes de la pandemia}

Según los datos de INEl la evolución de la población de la región Huancavelica es como muestra la tabla 1 , con una tasa de crecimiento promedio $1.3 \%$ anual y $0.26 \%$ de tasa de mortalidad promedio. El censo del 2017 (INEI, 2018) muestra que: la Región experimentó el mayor descenso poblacional en los últimos años, perdiendo aproximadamente el $28 \%$ de la población en comparación del año 2011, confirmando la tendencia migratoria durante los últimos años. Como refiere Izquierdo (2018) de la Universidad del Pacífico del Perú, "este último obedece a las condiciones económicas que ha experimentado la región en los últimos años, como es el escaso desarrollo económico y de servicios sociales".

\section{Tabla 1}

La población de la región Huancavelica

\begin{tabular}{ccccccccccc}
\hline Año & 2011 & 2012 & 2013 & 2014 & 2015 & 2016 & 2017 & 2018 & 2019 \\
\cline { 2 - 9 } \\
$\begin{array}{l}\text { Población } \\
\text { (personas) }\end{array}$ & 479641 & 453580 & 423472 & 390278 & 370963 & 353466 & 347639 & 371321 & 383200 \\
\hline
\end{tabular}

Nota. Tomado del Instituto Nacional de Estadística e informática (INEI 2018)

El pobre desarrollo económico en la Región ha tenido un débil impacto en la reducción de la pobreza. El INEI (2018) muestra la pobreza que bordea el 39\% de la población total; y Fatule (2020) en su informe de CEPAL, indica que: la pobreza para el año 2019 alcanzaba una disminución a 36.9\% y pobreza extrema 6.9\%. Según INEI (2018), estas condiciones desfavorables han promovido la migración a otras regiones del 
país, la emigración promedio anual desde el año 2011 al 2017 alcanzó aproximadamente el 6\% de la población total, las preferencias migratorias fueron las regiones Lima y Junín

Asimismo, en la tabla 2, según el INEI 2011 la Población Económicamente Activa (PEA) en región Huancavelica fue 298,700 personas que representaba al $62 \%$ de la población total. De estos, el $97.3 \%$ estuvieron ocupadas y el $2.7 \%$ desocupadas representando a 8065 personas sin empleo. El año 2017 la PEA registrada alcanzó un crecimiento de $2.8 \%$ llegando al $64.8 \%$, con una tasa de crecimiento promedio anual $1.7 \%$ en ese periodo y la PEA ocupada alcanzó la tasa de 1,6\%, lo que significa que cada año la población ocupada se ha incrementado en mil personas. Finalmente, el año 2011 el 67\% de la PEA se dedicaban a las actividades relacionadas con la agricultura en toda la región, y para el año 2017 tuvo una reducción de 1.7\% llegando a 65.3\% de la población total, siendo 199243 personas empleados en las actividades agrícolas.

\section{Tabla 2}

Evolución de la PEA

\begin{tabular}{llllllll}
\hline \multicolumn{1}{c}{ Año } & $\mathbf{2 0 1 1}$ & $\mathbf{2 0 1 2}$ & $\mathbf{2 0 1 3}$ & $\mathbf{2 0 1 4}$ & $\mathbf{2 0 1 5}$ & $\mathbf{2 0 1 6}$ & $\mathbf{2 0 1 7}$ \\
\cline { 2 - 8 } PEA & $62 \%$ & - & $52 \%$ & $53 \%$ & $53 \%$ & $75 \%$ & $64.8 \%$ \\
PEA ocupada & $97.3 \%$ & $98.1 \%$ & $98 \%$ & $98.7 \%$ & $99.5 \%$ & $98.8 \%$ & $97.6 \%$ \\
PEA desocupada & $2.7 \%$ & $1.9 \%$ & $2 \%$ & $1.3 \%$ & $0.5 \%$ & $1.2 \%$ & $2.4 \%$ \\
PEA agrícola & $67,0 \%$ & $68,7 \%$ & $65,6 \%$ & $71,8 \%$ & $66,4 \%$ & $65,2 \%$ & $65,3 \%$ \\
\hline
\end{tabular}

Nota. Tomado del Instituto Nacional de Estadística e informática INEI $(2011,2017,2018)$.

Huancavelica se caracteriza por ser una región altamente agrícola, donde más de $65 \%$ de la población se emplean en estas actividades, los productos con mayor superficie en las intenciones de siembra es la papa, la cebada grano y el maíz amiláceo. Según el Plan estratégico de la Gerencia Desarrollo Económico Regional (Región Huancavelica, 2009), "la producción total de la papa en la región alcanzó 161808 Tn en el año 2008; y el 2009 ya alcanzaba a 161,886.90 Tn. Así mismo, en el 2018 entre enero a marzo había alcanzado 44, 849 Tn". Y en el año 2019 para el Ministerio de Agricultura y Riego (Minagri, 2019), la producción de la papa solo durante ese periodo superó las $48 \mathrm{mil} \mathrm{Tn}$. El tubérculo tiene como destino: mercado local, donde el consumo per cápita anual en los últimos años aumentó hasta 89 kilos, según información del Minagri. Y al mercado internacional, como refiere Tinoco (2020) de la Agencia Andina Noticias, "el Perú exporta 500 Tn de la papa nativa anualmente, de los cuales los productores de región exportaron aproximadamente 200 Tn de la papa al año que representa el 0,123\% de la producción total”. Y el precio promedio del tubérculo alcanza S/. 0.95 soles por kilogramo siendo 950 soles la tonelada.

El otro producto con mayor producción es el maíz, según el Plan estratégico de la Gerencia Desarrollo Económico Regional (GDER), en el año 2008 alcanzó 19735 Tn y en el año 2009 alcanzaba 23,371 Tn. La 
demanda en el mercado local ha incrementado, según la Asociación de Gremios Productores Agrarios del Perú (2018), "el consumo per cápita de maíz amiláceo en nuestro país en los últimos quince años casi se duplicó, al pasar de 6.8 kilos a 12.3 kilos por persona al año". A nivel de la región, según el reporte de comercio regional de Huancavelica, la producción del maíz alcanza aproximadamente 27 mil Tn anuales, con el precio promedio de S/. 1.13 soles por kg siendo 1130 soles la Tn.

Y la producción de cebada en la región, según los datos del Ministerio de Agricultura (Minagri) en el año 2008 alcanzó 22441 Tn; para el año 2009 había alcanzado 26,847 Tn, comercializando el kilogramo del producto a un precio promedio de S/. 1.5 soles.

\section{Tabla 3}

Evolución de la producción agrícola

\begin{tabular}{|c|c|c|c|c|c|c|c|c|}
\hline Año & 2008 & 2009 & 2014 & 2015 & 2016 & 2017 & 2018 & 2019 \\
\hline $\begin{array}{l}\text { Prod. Papa } \\
\text { TM }\end{array}$ & 161808 & 161886.9 & 162986,9 & 163881,2 & 165816,9 & 176871,0 & 199396,0 & 211000,0 \\
\hline $\begin{array}{l}\text { Prod. Maíz } \\
\text { TM }\end{array}$ & 19735.0 & 23371.0 & 23871,0 & 24381,0 & 25376,0 & 27003,0 & 27432,0 & 29450,0 \\
\hline $\begin{array}{l}\text { Prod. } \\
\text { Cebada TM }\end{array}$ & 22441,0 & 26847,0 & 26947,0 & 27877,0 & 27947,0 & 28999,0 & 29200,0 & 50321,0 \\
\hline
\end{tabular}

Nota. Tomado del Ministerio de Agricultura Minagri (2019).

\section{Situación generada por la pandemia del covid-19}

El covid-19 tuvo efectos negativos en la economía de la población rural como de la región Huancavelica, paralizándose las actividades y muchas de ellas han sido restringidas en su cadena de comercialización. Como refiere (Prensa Latina, 2020). estos tendrían como efecto, ampliar la pobreza y la brecha de desigualdad entre el campo y la ciudad, según confirmó una encuesta del Instituto de Estudios Peruanos (IEP).

Vadillo (2020) del Ministerio de Agricultura y Riego estima: que el estado de emergencia viene afectando a la producción de la papa reduciendo en $1.8 \%$ la producción del año 2019". Debido a la poca demanda de la papa en los hoteles, los restaurantes y pollerías, que dejaron de funcionar como consecuencia de las restricciones por la emergencia sanitaria. Y los precios como indica Zurita (2020) "se han derrumbado hasta $-40 \%$ que van de S/. 0.30 a S/0.50 el kilo en la chacra. Asimismo, los efectos se aprecian en el comportamiento de precio de los cereales como maíz, trigo, cebada, etc. Según el Consejo Internacional de Granos, la proyección para la producción de cereales en la campaña 2020/21 se ha recortado en 5 millones de TM, ya que las perspectivas de cosecha han sido afectadas por condiciones atmosféricas 
desfavorables en Europa (Mercados \& Regiones, 2020). En cuanto a la producción de maíz, el USDA (United States Department of Agriculture) proyecta caídas -19.6\% hacia el año 2021.

Por otro lado, según la Radio Programa de Noticias (RPP) la pandemia tuvo efectos en el empleo de la población, como informa el INEI, que, en el segundo trimestre del 2020, la población ocupada del área rural totalizó 3 millones 524 mil 700 personas y al compararlo con el segundo trimestre del año pasado, se redujo en $-6,5 \%$ (-245 mil 500 personas) (RPP, 2020). Estos indican el incremento de desempleo, empeorando el nivel de la pobreza, que subiría entre $8 \%$ y $10 \%$ a fines de año, según proyecciones preliminares del Poder Ejecutivo, pasaría del 20.2\% registrado en el 2019 a un 30\%. Esta crisis económica, lo llevaría al país retroceder unos 10 años de lucha contra esta situación de vulnerabilidad.

\section{Diagrama de influencias}

Con la información obtenida previamente, se construyó el diagrama de la figura 1, donde se observan las variables y sus interrelaciones que pertenecen al problema de nuestro interés, que conforman un bucle de realimentación positiva. Este último ilustra la estructura sistémica y dinámica de efecto del covid-19 en la economía nacional.

La figura 1, nos permite realizar una descripción de la estructura dinámica del efecto del covid-19 en la economía. Donde el confinamiento social genera de manera positiva paralización de economía nacional. A medida que la economía permanece paralizada en el tiempo, provoca un aumento en la población desempleada. Estas necesidades, entre otras, permitieron a la población incumplir las medidas implementadas por el gobierno, provocando el aumento de comercio informal y desplazamiento de personas a los lugares de origen, generando el incremento de personas contagiadas por covid-19. El resultado de ello ha permitido continuar con el confinamiento social.

\section{Figura 1}

La estructura general del problema

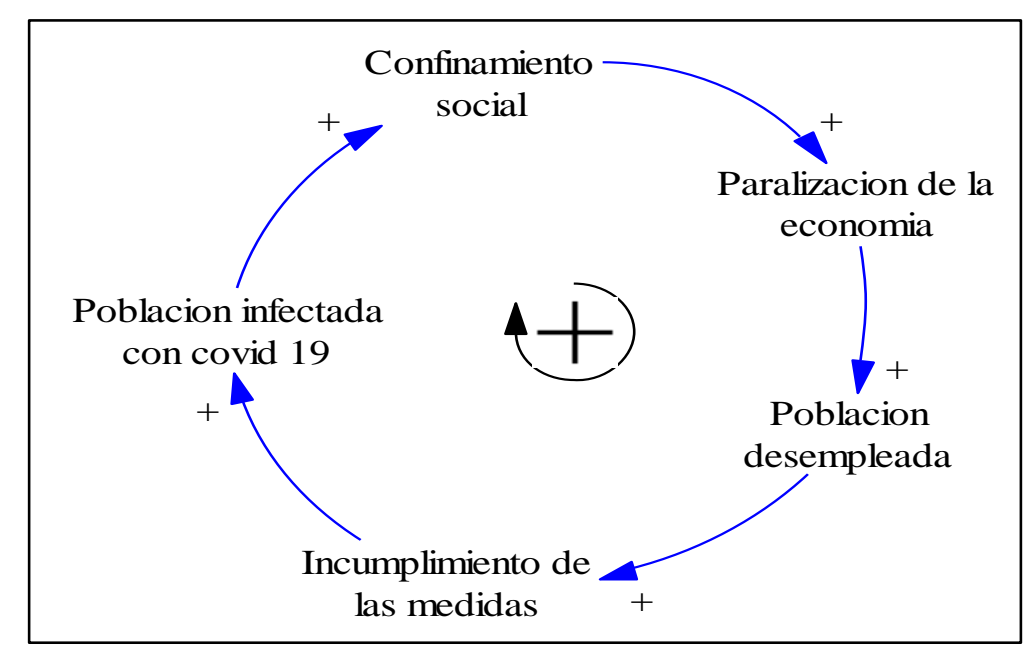


La figura 2, representa la problemática de la economía de la población rural a consecuencia del covid-19, formando tres bucles principales. Primero, el bucle positivo representa los efectos del confinamiento social en la disminución de la demanda de los productos agrícolas, que ha provocado bajar los precios de los productos a fin de contrarrestar su acumulación, afectando los ingresos y la utilidad anual de los agricultores. La reducción de utilidades interrumpe la actividad productiva, generando desabastecimiento de mercados locales y nacionales, permitiendo la elevación de precio de los productos que, afecta la economía de la población, principalmente a los más pobres. Lo anterior ha provocado en la población incumplir las acciones del gobierno, generando el aumento de los casos de covid-19. Segundo, el bucle negativo representa los efectos de desaceleración de la producción agrícola en el proceso de agroexportación, como consecuencia de reducción de la demanda de productos agrícolas en los países como China y EE.UU. provocando caída del PBI agrario y pérdida de empleos en las ciudades, generando el retorno de la población a sus lugares de origen, empeorando aún más la situación de pobreza y desigualdad en las zonas rurales, esto generaría de nuevamente la migración a las ciudades en busca de mejores oportunidades, abandonando el campo y la actividad agrícola. Y tercero, el bucle positivo representa los efectos de desaceleración de la producción agrícola en el desempleo rural, la misma empeorando aún más la situación de pobreza y desigualdad.

La formación de bucles entre las variables provoca comportamientos emergentes en el sistema, que son propiedades inherentes de los sistemas dinámicos, que, habitualmente no consideradas por tomadores de decisión, donde los bucles positivos provocan en el sistema una inestabilidad interna entre las variables. Y los bucles negativos generan una estabilidad interna. Estos explican, las variaciones provocados en una de las variables influirá en el resto recibiendo al mismo tiempo un impacto de su propia variación. 


\section{Figura 2}

Diagrama de la problemática de economía rural como efecto de la covid-19

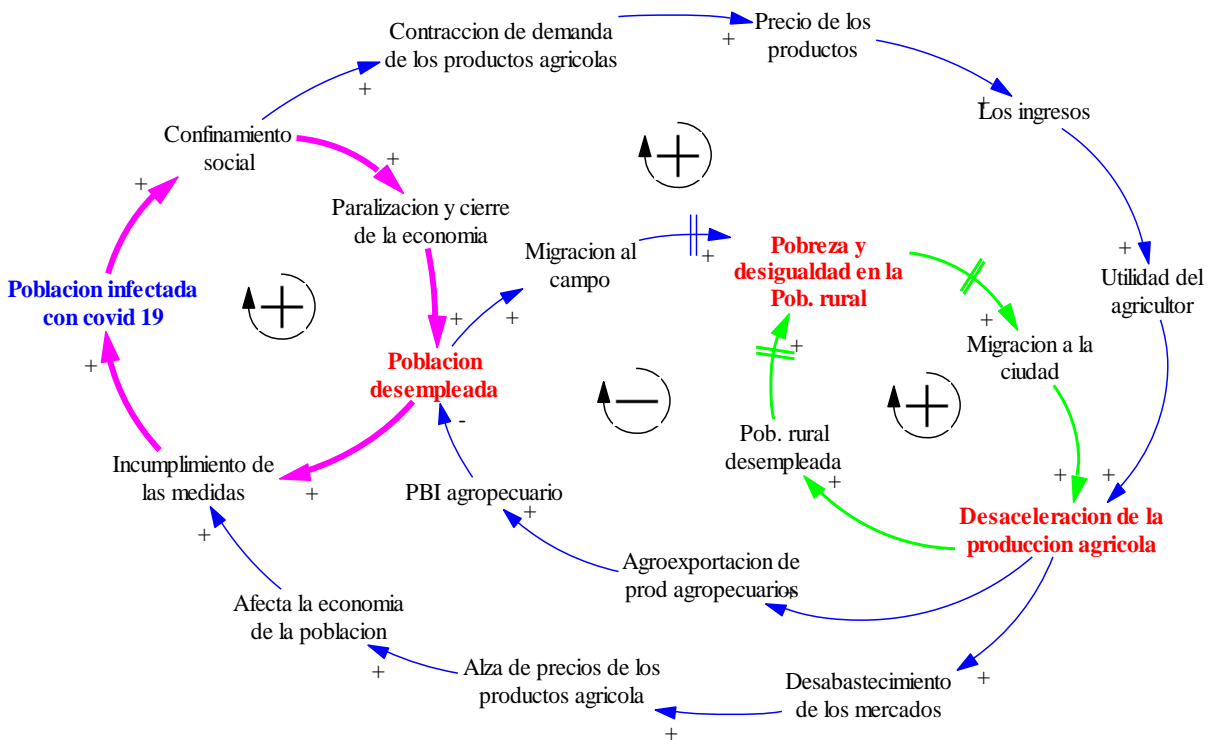

En la figura 2, se puede percibir dos variables exógenas, la pandemia del covid-19, que ha puesto en una situación inestable el sistema económico de la población. Y la globalización económica, como factor causante de la velocidad y la manera en la que el covid-19 se propagó por todos los rincones del planeta, como característica de la era de mayor interconexión mundial en la historia de la humanidad. Mizrahi (2020), refiere "sin los flujos inéditos de personas, mercancías e información que caracterizan a esta era, sería imposible un brote de estas proporciones".

\section{Modelo de simulación}

Para observar la evolución dinámica de las variables relevantes de nuestra situación problemática, se ha traducido el diagrama de influencias con ayuda del software de simulación Vensim a un diagrama de Forrester, una terminología que permite mostrar la estructura de las ecuaciones en el ordenador, como se aprecia en la figura 3. Donde un principal bucle de realimentación resaltado con un color distinto, indica, las modificaciones provocados en una de las variables influirá en el resto de las variables del sistema. El bucle indica, cómo el normal comportamiento de la influencia entre las variables de población, la demanda de los productos, la producción, la utilidad de los agricultores y condiciones económicas, vienen siendo afectada por las variables exógenas. Afectando directamente a la demanda y precio de los productos agrarios. 


\section{Figura 3}

Diagrama de Forrester de la problemática de economía como efecto del covid-19

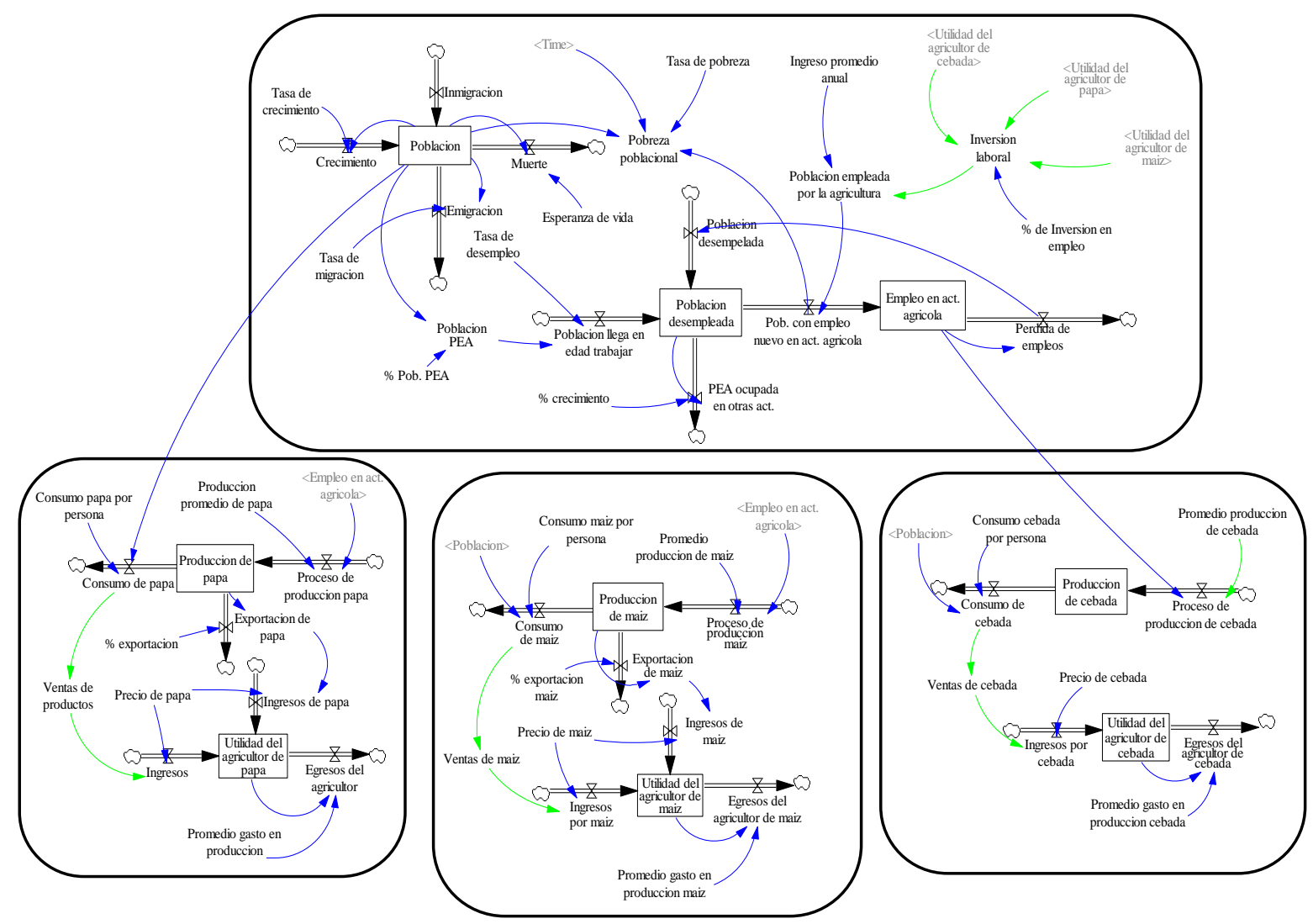

\section{Validación del modelo}

En este apartado se presenta el resumen de la validación del modelo desarrollado, tomando referencia John Sterman (2000), algunos test de análisis estructural y comportamiento, recogidos y sintetizados (p. 843), con la que se demuestra que el modelo dinámico presenta un nivel de relevancia, demostrando su confiabilidad y la calidad del mismo.

Primero, en aplicación de la técnica de análisis de estructura, el modelo fue sometido al test verificación de la estructura del modelo, que responde la interrogante ¿Es la estructura del modelo consistente con el conocimiento descriptivo relevante del sistema? Consiste en comparar la estructura del modelo con la estructura del sistema real que el modelo representa. En el desarrollo del modelo, se realizó una revisión detallada de literaturas referidas al impacto del covid-19 en la agricultura, el empleo y la pobreza; tomando en cuenta sus aportes y enfoques utilizadas. Además, se examinó la literatura en el desarrollo de modelos como Industrial Dynamics y Business Dynamics; de los de los autores J. Forrester, J. Aracil y J. Sterman, respectivamente. Como principales fuentes de información y representa el origen y las bases en materia del desarrollo del modelo. 
Con el test de verificación de parámetros, responde la interrogante ¿Son los parámetros consistentes con el conocimiento descriptivo relevante del sistema? Implica comparar los parámetros del modelo con el conocimiento disponible sobre el sistema real. Por lo tanto, los valores de parámetros que condicionan el comportamiento del modelo desarrollado tuvieron como fuentes las instituciones como, el INEI proporcionando los Indicadores de empleo e ingreso por región, periodo 2007-2017. También se utilizó la información de la agricultura en tiempos de la pandemia proporcionada por el PNUD. La CEPAL proporcionó información respecto de los efectos del covid-19 que tendrá sobre la economía mundial y sus efectos en la América Latina. Y finalmente el MINAGRI y DRA Huancavelica contribuyó con las informaciones de la producción agrícola en la Región.

Y el Test de Consistencia Dimensional, responde la interrogante ¿Son todas las ecuaciones del modelo dimensionalmente consistentes sin necesidad de utilizar parámetros sin significado real en términos del problema? implica un análisis dimensional de las tasas incluidos en el modelo, comprobando de este modo que, todas y cada una de las variables que lo conforman, aparecen expresadas en las unidades de medida adecuadas. La consistencia de dimensiones que conforman las ecuaciones del modelo desarrollado, fueron verificadas con la ayuda de software Vensim que cuenta con las herramientas Check Model y Units Check. Y, por último, se verificó los valores asignados a los parámetros, con fuentes proporcionadas de las entidades involucradas, así como el INEI.

Segundo, el modelo ha sido preparado para mostrar los estados del sistema desde 2011 al 2026. El período comprendido entre 2011 hasta el 2019, ha sido para corroborar el funcionamiento del modelo. Por eso, a través de la técnica análisis de comportamiento el modelo fue sometido al Test de Comportamiento Estadístico que responde a la interrogante ¿Tiene el comportamiento del modelo las mismas características estadísticas que el comportamiento del sistema real? Que implica comparar la estructura del modelo directamente a los datos empíricos disponibles. Donde se evaluó el desempeño del modelo, analizando el porcentaje de error generado de los datos simulados y datos reales. Tomando como referencia el estudio de Barlas (1996), que establece que "un modelo será válido si la tasa de error es más pequeña que el 5\%". Tomando en consideración, expresión matemática que establece la ecuación siguiente:

$$
\% \text { error relativo }=\frac{\text { dato simulado }- \text { dato real }}{\text { dato real }} * 100
$$

Las variables representativas, sometidas a la evaluación fueron: el nivel de la rentabilidad de los productores de la papa, el maíz y la cebada, y el empleo en las actividades relacionados con la agricultura. 
La tabla 4, resume los resultados de la técnica de validación del comportamiento. Los análisis de los datos de las variables sometidas al análisis determinan que, la media obtenida entre los datos reales y simulados, presentan medias aproximadas con variación mínima, menores del 5\%.

Tabla 4

Resumen de comportamiento de datos simulados y datos reales

\begin{tabular}{lcccc}
\hline & Pob. & Prod. de papa & Prod. de maíz & Prod. cebada \\
\cline { 2 - 4 } Datos Estadístico & & & & \\
Media & 397951 & 173948,2 & 24887,7 & 34380,6 \\
Desviación estándar & 44584 & 18557 & 2838,1 & 7975,8 \\
Mínimo & 353466 & 161808 & 19735 & 22441 \\
Máximo & 479641 & 211000 & 29450 & 50321 \\
Nivel de confianza (95\%) & 34270 & 14264,2 & 2181,6 & 6130,7 \\
Datos Simulados & & & & 35720,1 \\
Media & 402193 & 177598,3 & 25789,7 & 5568,9 \\
Desviación estándar & 42730 & 28462,4 & 2864.0 & 26847.0 \\
Mínimo & 362238 & 161886 & 23371.0 & 42475,5 \\
Máximo & 479641 & 241019 & 31335,1 & 4280,6 \\
Nivel de confianza (95\%) & 32845 & 21878,1 & 2201,4 & $3,90 \%$ \\
Error relativo de datos & $1,07 \%$ & $2,10 \%$ & $3,62 \%$ & \\
\hline
\end{tabular}

Nota. Procesamiento de validación del modelo obtenidas con SPSS v23.

Y el Test de Sensibilidad del Comportamiento, responde la interrogante ¿Es el modelo sensible a variaciones plausibles de los parámetros? Que implica en centrarse su atención en la sensibilidad del modelo ante cambios en el valor de los parámetros. Para comprobar se realizaron simulaciones con ayuda de software Vensim, donde el rango de incertidumbre de parámetros fue el $25 \%$ del valor real como muestra la tabla 5 .

\section{Tabla 5}

Rango de incertidumbre de parámetros

\begin{tabular}{|c|c|c|c|c|}
\hline \multirow[b]{2}{*}{ Parámetros } & \multirow[b]{2}{*}{ Unidad } & \multirow[b]{2}{*}{$\begin{array}{l}\text { Valor } \\
\text { real }\end{array}$} & \multicolumn{2}{|c|}{ Rango de incertidumbre } \\
\hline & & & $\begin{array}{c}\text { Valor menor de } \\
-25 \%\end{array}$ & $\begin{array}{c}\text { Valor mayor de } \\
+\mathbf{2 5 \%}\end{array}$ \\
\hline Consumo de la papa & Toneladas/(Personas*Year) & 0.089 & 0.067 & 0.111 \\
\hline Precio de la papa & Soles/Toneladas & 950 & 712.5 & 1187.5 \\
\hline Consumo del maíz & Toneladas/(Personas*Year) & 0.0123 & 0.009 & 0.0153 \\
\hline Precio del maíz & Soles/Toneladas & 1130 & 847.5 & 1412.5 \\
\hline Consumo de cebada & Toneladas/Personas/Year & 0.015 & 0.012 & 0.018 \\
\hline Precio de la cebada & Soles/Toneladas & 1500 & 1125 & 1875 \\
\hline
\end{tabular}


Las figuras de 4 al 5, muestran algunos resultados obtenidos de la variación de parámetros inciertos en un rango de incertidumbre al $25 \%$ del valor real y así como, la simulación base. Donde, si consideramos una variación $+25 \%$ del valor real, los valores de las variables relevantes al 2019 presentan una tendencia menor que la simulación base. Sin embargo, una variación -25\% del valor real, los valores de las variables al 2019 presentan una tendencia mayor que la simulación base, los resultados se aprecian en la 6. Por lo que, el comportamiento de las variables bajo las condiciones evaluadas, presentan un comportamiento aceptable.

\section{Tabla 6}

Rango de variación de valores de las variables

\begin{tabular}{lccccc}
\hline \multirow{2}{*}{$\begin{array}{c}\text { Parámetros del } \\
\text { modelo }\end{array}$} & \multicolumn{4}{c}{ Las variables } \\
\cline { 2 - 5 } & Utilidad de prod. & Utilidad de & Utilidad de & Nivel empleo en las \\
papa & prod. Maíz & prod. cebada & act. de agrícola \\
\hline \multirow{2}{*}{ Valores } & $-25 \%$ & 60000000 & 37500000 & 16000000 & 203200 \\
del rango & $100 \%$ & 138310000 & 51336100 & 37210700 & 205527 \\
& $+25 \%$ & 224000000 & 67500000 & 64000000 & 208000 \\
\hline
\end{tabular}

Nota. Valores determinados en software Vensim.

En conclusión, el modelo desarrollado presenta el nivel de confiabilidad aceptable que responde positivamente los propósitos del desarrollo. Por lo que es empleado para analizar las variables de interés y las políticas establecidas.

\section{Figura 4}

Análisis de sensibilidad de la utilidad en producción de la papa

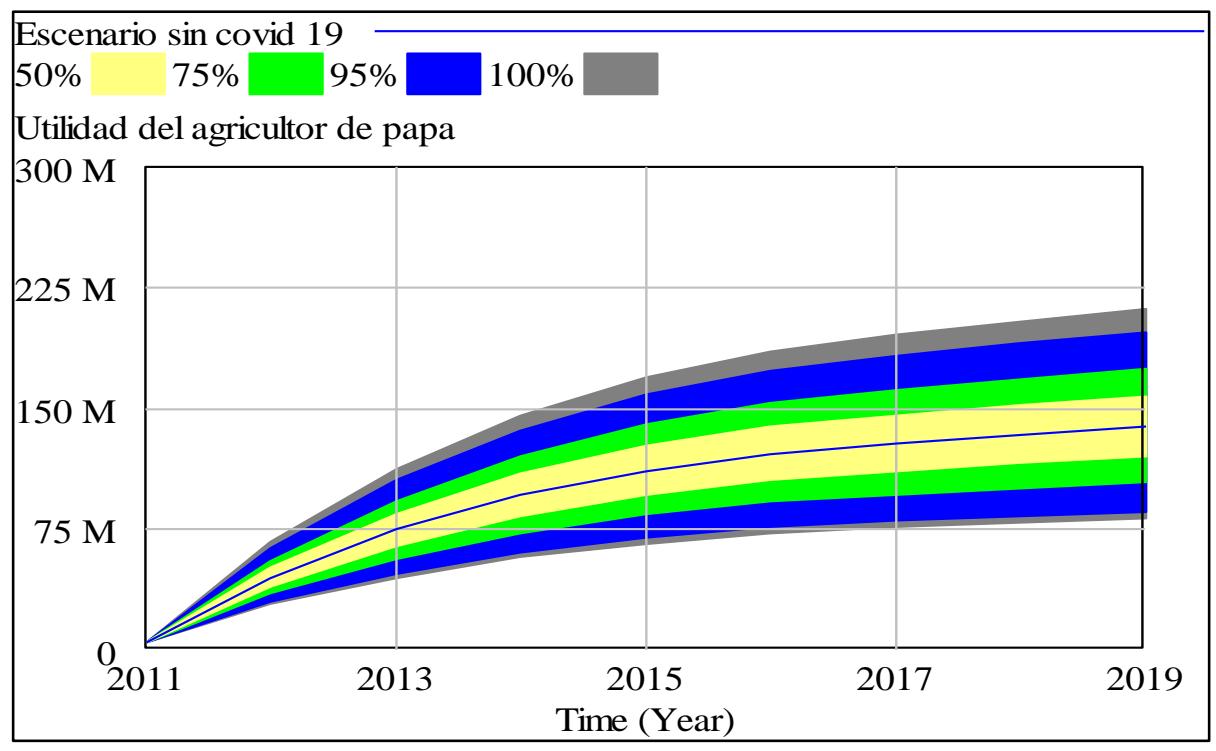

Nota. Análisis desarrollado en software Vensim. 


\section{Figura 5}

Análisis de sensibilidad del empleo en las actividades agrícolas

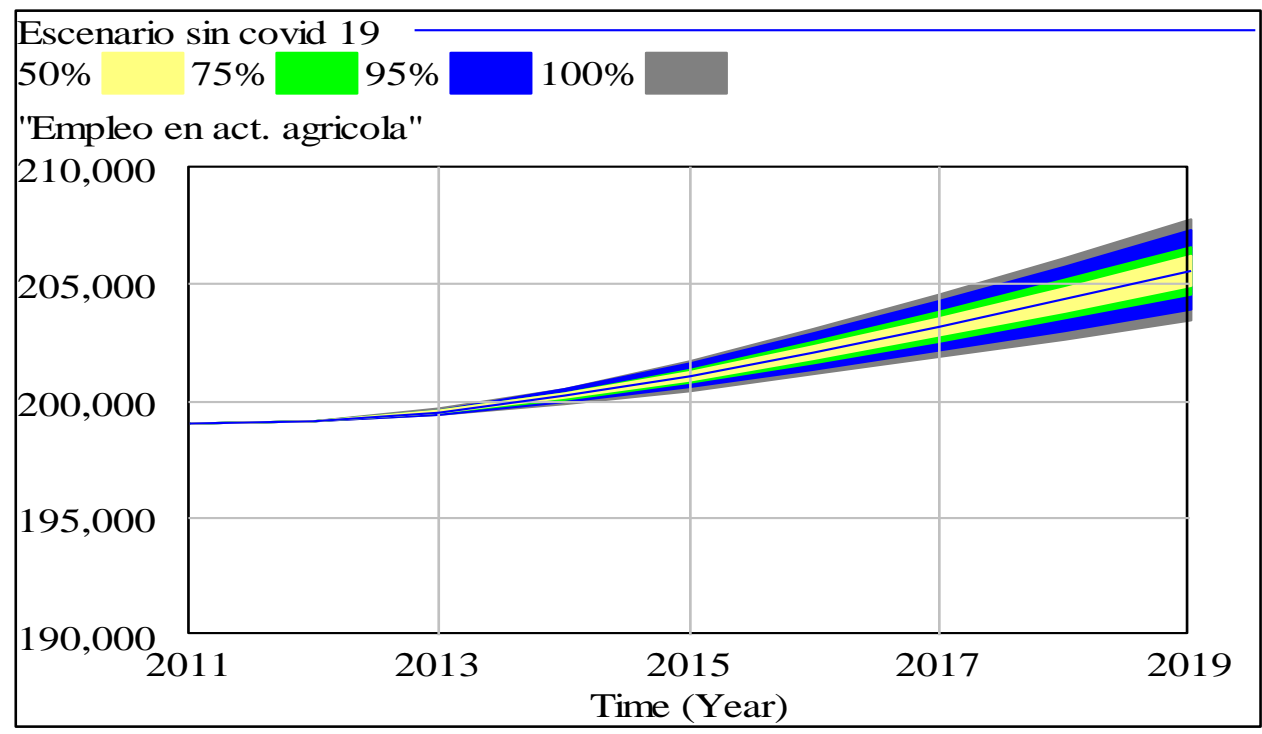

Nota. Análisis desarrollado en software Vensim.

\section{Resultado}

Para comprender el efecto de la pandemia del covid-19 en la economía de población a través el modelo desarrollado, se presentan tres escenarios que fueron analizados en un periodo entre 2011 hasta el 2026. El primer escenario analiza el comportamiento de las variables en una situación normal, sin covid-19. El segundo escenario, analiza la situación generada por covid-19 con efectos negativos en la economía de la población. Y el tercero evalúa la economía de la población con los mecanismos dados por el gobierno para mejorar la situación generada por la pandemia. Los valores de los parámetros y condiciones iniciales seleccionados para la simulación de los escenarios se presentan la tabla 7. 
Tabla 7

Valores de parámetros en los tres escenarios

\begin{tabular}{|c|c|c|c|c|c|c|}
\hline Parámetros & Unidad & $\begin{array}{c}\text { Escenario } \\
01\end{array}$ & $\begin{array}{c}\% \\
\text { Variación }\end{array}$ & $\begin{array}{l}\text { Escenario } \\
02\end{array}$ & $\begin{array}{c}\% \\
\text { Variación }\end{array}$ & $\begin{array}{c}\text { Escenario } \\
03\end{array}$ \\
\hline Consumo de la papa & $\begin{array}{c}\text { Toneladas/(Personas*Y } \\
\text { ear) }\end{array}$ & 0.089 & $-13 \%$ & 0,08 & $+3,30 \%$ & 0,092 \\
\hline Precio de la papa & Soles/Toneladas & 950 & $-40 \%$ & 570,00 & $+100 \%$ & 1900 \\
\hline Consumo del maíz & $\begin{array}{c}\text { Toneladas/(Personas*Y } \\
\text { ear) }\end{array}$ & 0.0123 & $-13 \%$ & 0,01 & $+3,30 \%$ & 0,013 \\
\hline Precio del maíz & Soles/Toneladas & 1130 & $-40 \%$ & 678,00 & $+100 \%$ & 2260 \\
\hline Consumo de cebada & $\begin{array}{c}\text { Toneladas/Personas/Ye } \\
\text { ar }\end{array}$ & 0.015 & $-13 \%$ & 0,01 & $+3,30 \%$ & 0,019 \\
\hline Precio de la cebada & Soles/Toneladas & 1500 & $-40 \%$ & 900,00 & $+100 \%$ & 3000 \\
\hline Tasa de pobreza & 1/año & $36.9 \%$ & $+10 \%$ & $40,59 \%$ & - & - \\
\hline Personas con empleo & Personas & $65.3 \%$ & $-10 \%$ & $58,77 \%$ & - & - \\
\hline
\end{tabular}

Nota. Elaborado con los datos proporcionados por Instituto Nacional de Estadística e informática INEI, Programa de las Naciones Unidas para el Desarrollo PNDU, Ministerio de Agricultura MINAGRI, Cámara de Comercio de Lima CCL.

\section{Escenario 01 situación normal}

Los resultados se muestran en la figura 6, cuando el consumo per cápita y el precio de los productos agrarios continúan tomando los valores actuales, el comportamiento de la producción de la papa, el maíz y la cebada, tendrían una tendencia ascendente para los próximos años, y la utilidad de los agricultores seguiría la misma tendencia. Estos favorecen al crecimiento del empleo en las actividades agrícolas. Se estima 215342 personas con empleo al año 2026, el 4.7\% más del año 2019. Sin embargo, las personas en condiciones de pobreza continuarían con su crecimiento, llegando al año 2026 con 153047 personas en situación de pobreza, que sería 12.3\% más del año 2019.

\section{Escenario 02 situación del covid-19}

Escenario que corresponde a la situación de la pandemia que se produce durante el año 2020 y sus efectos negativos en la economía de la población, se muestra en la figura 7. Debido a la caída del consumo de productos agrarios hasta $-13 \%$ de la producción normal; así como, de los precios hasta $-40 \%$, afectando negativamente a la producción de las actividades agrícolas. Esto ha provocado la caída del empleo hasta menos $-10 \%$, empeorando la situación de la pobreza con un crecimiento hasta $+10 \%$ en comparación de los años anteriores. Además, se debe a la migración durante la pandemia, de la ciudad al campo, migrando 20000 personas, aproximadamente, a la región Huancavelica. 
Si esta situación continua hacia los próximos años, la tendencia de la producción de la papa, el maíz y la cebada, tendrían una caída continua. Como consecuencia, las utilidades de los agricultores también presentarían una caída. La utilidad de los productores de la papa alcanzaría el 33\% menos que en la situación normal. De productores del maíz hasta menos 33.2\%. Y de productores de la cebada sufrirían hasta menos de 41\%. Estos provocarían la caída del empleo, llegando para el año 2026 con 193249 personas sin empleo, el 10\% menor que la situación normal. La pobreza alcanzaría 168587 personas en situación de pobreza para el año 2026, el 16\% mayor que la situación normal.

\section{Escenario 03, mecanismos para mejorar los efectos del covid-19}

Escenario que corresponde a la situación donde el gobierno y la población, buscan mecanismos para reactivar la agricultura. Por un lado, el Ministerio de Agricultura y Riego, a través de Agro Rural ha establecido mecanismos que busca aumentar el consumo de la papa en las grandes ciudades del Perú. Vadillo (2020) refiere, el mecanismo busca crear una alianza estratégica con una cadena de supermercados y promocionar a través Adopta tu tonelada de papa. Con esta intervención el estado busca paliar la reducción del consumo negativo de papa que se registró durante los meses de la coyuntura del covid-19, cuando los hoteles, los restaurantes y pollerías dejaron de funcionar. Intervenciones que permitirán a 711 mil familias de 19 regiones del Perú, dedicadas a la producción de papa, tener canales de comercialización que garantice el aumento de la utilidad de los agricultores y afrontar esta emergencia del covid-19.

Por otro lado, como refiere Vadillo (2020), otros mecanismos importantes para incrementar el consumo de productos agrarios como la papa, el maíz, la cebada entre otros, fueron los mercados itinerantes más conocida como De la Chacra a la olla, que consiste en organizar ferias en grandes ciudades, donde los productos son llevados directamente de la chacra. Que presenta una gran ventaja para los productores, que ofrecen el $\mathrm{Kg}$. de productos en un precio mayor de $100 \%$ que vendían sus productos a intermediarios. Estos mecanismos permitirían aumentar el consumo per cápita de la papa a 92 kilos para el 2021 con un crecimiento de 3.3\%, en comparación de años anteriores que era 89 kilos, el porcentaje estimado para los demás productos agrarios.

Las tendencias de las variables hacia los próximos años, como resultado de los mecanismos implementados que muestran la figura 8. Al año 2026 las utilidades de los productores de la papa incrementarían hasta más de $80 \%$ en comparación de la situación normal. De productores del maíz hasta más de $60 \%$. Y de la misma forma, de productores de cebada alcanzarían hasta más de $20 \%$. Sin embargo, estos no tendrían los resultados esperados en la economía de la población, toda vez que, el empleo en las actividades agrícolas lograría recuperarse solo el 1.8\% en comparación a la situación provocada por covid- 
19. Asimismo, las personas en pobreza solo disminuirían hasta $1 \%$. Quiere decir, los mecanismos dados por el Ministerio de Agricultura y Riego, a través de Agro Rural no tendrían efectos relevantes en la economía de la población, situación que requiere otras estrategias más eficientes y sostenibles, para recuperar el empleo perdido a causa de la pandemia y reducir alto porcentaje de la pobreza en la región Huancavelica.

\section{Figura 6}

Tendencias de las variables en el escenario normal



Nota. Resultados de análisis obtenida con software Vensim.

\section{Figura 7}

Tendencias de las variables en el escenario del covid-19

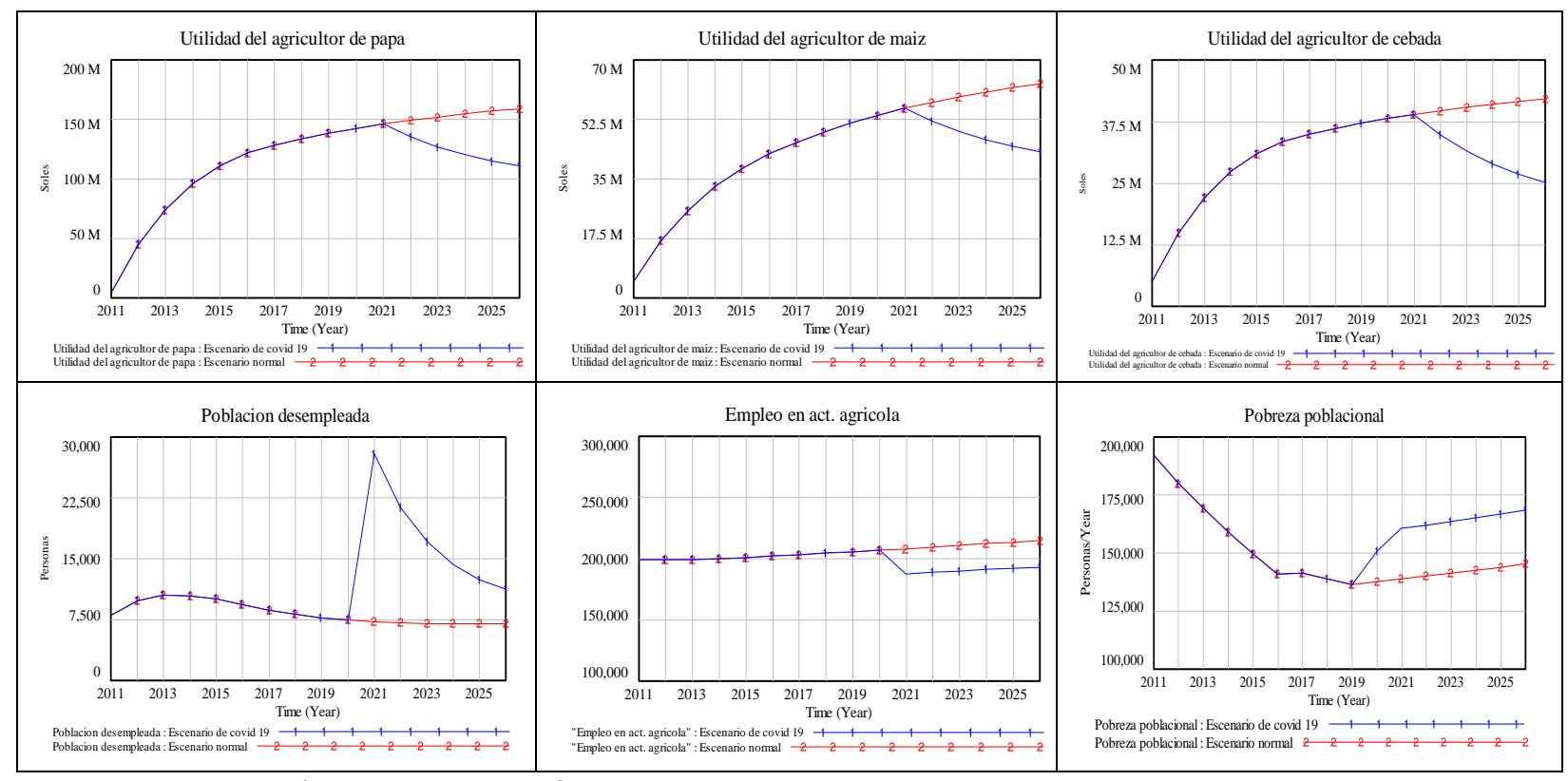

Nota. Resultados de análisis obtenida con software Vensim. 


\section{Figura 8}

Tendencias en el escenario con mecanismos para mejorar los efectos del covid-19

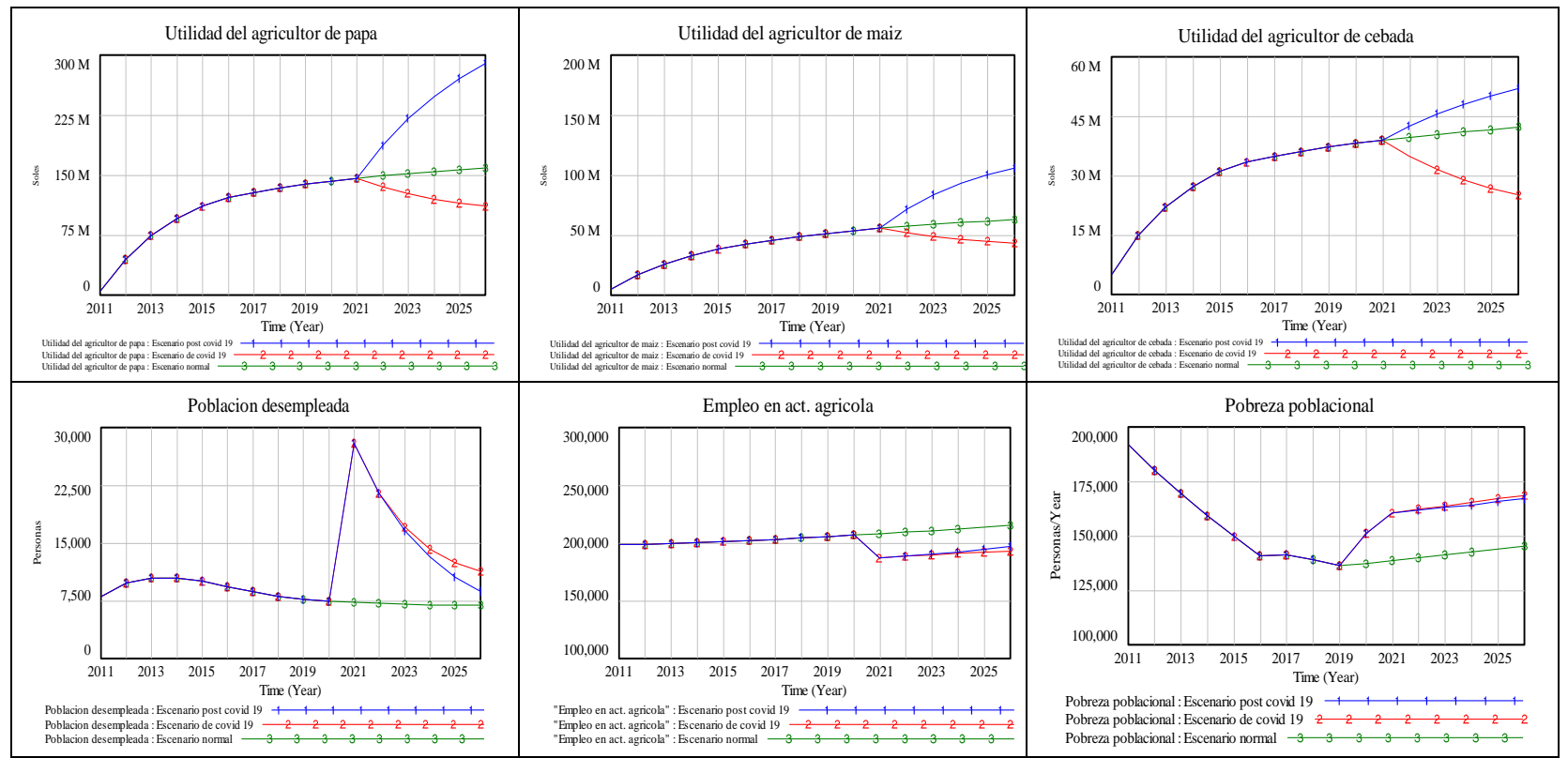

Nota. Resultados de análisis obtenida con software Vensim

\section{Discusión}

La restricción del acceso a mercado nacional e internacional, como consecuencia de las medidas adoptadas por los gobiernos para frenar los contagios del covid-19, generó la mayor contracción económica en las regiones del país, donde la demanda y precio de los productos de la papa, el maíz y la cebada, sufrieron una variación negativa. Cayendo aproximadamente el 13\% y 40\% durante los meses de la pandemia, respectivamente. Generando el decremento del empleo hasta el $10 \%$ y un incremento de niveles de pobreza hasta el 10\% en comparación del año 2019. Resultados que guardan una relación con los análisis realizados por Obregón (2020, párr. 5) de la Agencia Agraria de Noticias:

Llegado el covid-19 y al entrar a la emergencia nacional, los agricultores vieron la caída de precios y el frenazo total de la demanda para algunas variedades de la papa (...). La demanda cayó a cero y los precios de algunas variedades de la papa cayeron hasta $S / 0.40$ el kilo por mayor. Que históricamente solo bajó hasta los S/0.70 el kilo, provocando la pérdida de los productores.

Los resultados obtenidos también guardan relación a las conclusiones adquiridas por Arteaga (2020, p. 15) de la revista de La Cámara de Comercio de Lima: 
Los consumidores finales de los productos agrícolas tienen menos ingresos, como resultado de producto de los despidos laborales y dejan de consumir los productos alimenticios. Como resultado los precios de los productos disminuyen. Donde el ingreso de los agricultores cada vez es menor.

Las simulaciones para el año 2026, indican, si no se toma acciones urgentes al respecto, el porcentaje de empleo persistiría para el año 2026. Es más, los mecanismos dados por el gobierno, solo lograría recuperar el $1.8 \%$ de empleo, en comparación del escenario de covid-19. Así mismo, el nivel de la pobreza con los mecanismos dados por el gobierno solo lograría disminuir la pobreza en 1\%, en comparación del escenario de covid-19. Por tanto, la agricultura requiere otras medidas más eficientes para volver ser más competitivo y sostenible. Estos resultados aproximan a las estimaciones de Lavado (2020, p. 23) de Fondo de las Naciones Unidas para la Infancia UNICEF:

La pobreza monetaria se incrementará de $20.2 \%$ en el 2019 a 30.3\% en el 2020 . Esto significa que 3,300,329 personas caerán en la pobreza como consecuencia directa de la pandemia. Será el mayor valor registrado en este grupo desde el 2010. Asimismo, considerando la recuperación en el ingreso de los hogares, se estima que este nivel de pobreza se reduzca a $25.8 \%$ en el 2021.

Asimismo, los resultados de la investigación relacionados con los análisis realizados por Arteaga (2020, párr. 6) de la revista de La Cámara de Comercio de Lima:

La agricultura requiere mucho más que dinero para capital de trabajo, o préstamos baratos. Es indispensable que los haya, pero si queremos que el agro sea competitivo se debe apuntar a medidas más eficientes. Lo que le falta al sector es contar con más elementos en el plan de reactivación agraria planteado, para ser más competitivo.

\section{Conclusión}

Los resultados de la investigación evidencian que la pandemia del covid-19 tuvo efectos negativos en la economía de la población de región Huancavelica. Debido a la desaceleración de la producción agrícola, generada por la caída del nivel de consumo y el precio de productos agrarios, consecuentemente la disminución de la utilidad de los agricultores; generando caída del empleo y empeorando las condiciones de la pobreza en la región. Se debe, además, al proceso migratorio de la ciudad al campo.

Las tendencias de las simulaciones para el año 2026, indican, que la situación empeoraría si no se toma acciones eficientes. Donde la utilidad económica de los productores agrícolas caería hasta $-30 \%$, aproximadamente, en comparación a las tendencias de la situación normal. Asimismo, el empleo en las 
actividades agrícolas caería en 10\%, disminuyendo de 215170 a 193249 personas con empleo y el nivel de la pobreza aumentaría en 10\%, de 152996 a 168587 personas en estado de pobreza.

Las simulaciones para el año 2026, indican, que los mecanismos implementados por el Ministerio de Agricultura y Riego, con la finalidad de incrementar el consumo per cápita de los productos hasta 3.3\% y el precio de los productos hasta el 100\%, en comparación del año 2019. No tendrían resultados esperados, el empleo en las actividades agrícolas solo lograría recuperar-se en 1.8\%, aumentando de 193249 a 197 398 personas con empleo. Asimismo, los niveles de pobreza solo lograrían disminuir hasta $1 \%$, pasando de 168587 a 166876 personas en estado de pobreza.

\section{Referencias}

Alejandro Berrospi, J. (26 de marzo de 2020). Situación de la Agricultura Familiar frente al Covid-19.

Servindi. https://www.servindi.org/actualidad-noticias/26/03/2020/importancia-de-laagricultura-familiar-para-enfrentar-el-covid-19

Aracil, J. y Gordillo , F. (1998). Dinamica De Sistemas. Anzos.

Arteaga, E. (24 de agosto de 2020). Agro peruano: antes, durante y después de la pandemia. La Cámara. https://lacamara.pe/agro-peruano-antes-durante-y-despues-de-la-pandemia-covid-19/

Bárcena, A. (19 de marzo de 2020). COVID-19 tendrá graves efectos sobre la economía mundial e impactará a los países de América Latina y el Caribe. CEPAL. https://www.cepal.org/es/comunicados/covid-19-tendra-graves-efectos-la-economia-mundialimpactara-paises-america-latina

Barlas, Y. (1996). Formal aspects of model validity and validation in system dynamics. System Dynamics Review, 12(3), 14.

Berganza, I. (7 de julio de 2020). Nuestros migrantes internos y covid-19. Diario El Peruano. https://elperuano.pe/noticia-nuestros-migrantes-internos-y-covid19-99695.aspx

INEI. (14 de noviembre de 2018). Censos Nacionales 2017: XII de Población, VII de Vivienda y III de Comunidades Indígenas. INEI. http://censo2017.inei.gob.pe/en-huancavelica-se-censo-a-347639-

personas/\#: :text=Al\%20a\%C3\%B10\%202017\%2C\%20el\%20departamento,y\%20Ayacucho\%201 $2 \% 2 \mathrm{C} 5 \% 20 \% 25$. 
Lavado, P. (2020). COVID-19: Impacto en la pobreza y desigualdad en niñas, niños y adolescentes en el Perú, Estimaciones 2020-2021. UNICEF Perú.

León, M. (20 de abril de 2020). Proteger la agricultura en tiempos de pandemia. Medium. https://medium.com/@PNUDperu/proteger-la-agricultura-en-tiempos-de-pandemia$30 \mathrm{bb} 9775 \mathrm{cc} 5 \mathrm{e}$

Marco Vinelli , R. (24 de abril de 2020). Impacto del COVID-19 en el empleo en el Perú. ESAN. https://www.esan.edu.pe/conexion/actualidad/2020/04/24/impacto-del-covid-19-en-elempleo-en-el-peru/

Mercados \& Regiones . (19 de mayo de 2020). Precios de granos afectados por las medidas para contener la pandemia. Mercados y Regiones. https://mercadosyregiones.com/2020/05/19/precios-degranos-afectados-por-las-medidas-para-contener-la-pandemia/

Minagri. (30 de mayo de 2019). Trabajaremos para que las 711 mil familias productoras de papa de todo el país sean prósperas. Plataforma digital de Ministerio de Agricultura y Riego. https://www.gob.pe/institucion/minagri/noticias/28926-trabajaremos-para-que-las-711-milfamilias-productoras-de-papa-de-todo-el-pais-sean-prosperas

Mizrahi, D. (12 de abril de 2020). La pandemia de coronavirus es hija de la globalización: ¿será su salvadora o su sepulturera? Infobae. https://www.infobae.com/america/mundo/2020/04/12/lapandemia-de-coronavirus-es-hija-de-la-globalizacion-sera-su-salvadora-o-su-sepulturera/

Obregón, C. (01 de junio de 2020). Impacto del Covid-19 en el sector papero del Perú. Agencia Agraria de Noticias. https://agraria.pe/noticias/impacto-del-covid-19-en-el-sector-papero-del-peru-21645

Prensa Latina. (06 de junio de 2020). Elhambre aliado estrategico de la covid-19. Prensa Latina. https://www.prensa-latina.cu/index.php?o=rn\&id=372080\&SEO=el-hambre-aliado-estrategicode-la-covid-19-en-peru

Región Huancavelica. (2009). Plan Estratégico Regional del Sector Agrario de huancavelica 2009 - 2015 [Archivo PDF]. Autor.

RPP. (15 de agosto de 2020). Coronavirus en Perú, INEI más de 6 millones de personas dejaron de trabajar debido a la pandemia noticia. RRP Noticias. https://rpp.pe/economia/economia/coronavirus-enperu-inei-mas-de-6-millones-de-personas-dejaron-de-trabajar-debido-a-la-pandemia-noticia1286728 ?ref=rpp 
RPP. (3 de agosto de 2020). Suspensión perfecta de labores: Estos son los requisitos para cobrar bono de S/ 760. RRP Noticias. https://rpp.pe/economia/economia/suspension-perfecta-sepa-losrequisitos-para-cobrar-bono-de-s-760-para-trabajadores-noticia-

1284051?utm_source=siguientenota\&utm_medium=scroll\&utm_campaign=siguientenota_scroll

Sánchez Carlessi, H. y Reyes Meza, C. (2017). Metodologia y Diseños en la Investigación Científica. Rícardo Palma

Tinoco, P. (30 de mayo de 2020). Agencia Peruana de Noticias. Recuperado el 07 de julio de 2020, de https://andina.pe/agencia/noticia-dia-nacional-de-papa-peru-exporta-500-toneladas-papanativa-al-ano-711671.aspx

Vadillo Vila, J. (5 de mayo de 2020). Minagri mantiene meta de 92 kilos de consumo de papa por persona para 2020 y 2021. El diario oficial El Peruano. https://elperuano.pe/noticia-minagri-mantienemeta-92-kilos-consumo-papa-persona-para-2020-y-2021-96598.aspx

Zurita, M. (8 de mayo de 2020). ¿Por qué cayeron los precios del tubérculo a la mitad durante la cuarentena? Diario El Comercio. https://elcomercio.pe/economia/dia-1/produccion-de-papapor-que-cayeron-los-precios-del-tuberculo-a-la-mitad-durante-la-cuarentena-informe-noticia/

\section{Contribución de los autores}

SHL: Conceptualización, análisis formal, investigación, metodología, redacción: revisión y edición.

FSC: Conceptualización, análisis formal, investigación, metodología, redacción: borrador original.

\section{Fuentes de financiamiento}

Autofinanciado.

\section{Conflictos de interés}

No presenta conflicto de intereses.

\section{Correspondencia}

sergio.huincho@unh.edu.pe 